\title{
Direct and Homogeneous Numerical Approaches to Multiphase Flows and Applications
}

\author{
Roman Samulyak ${ }^{1}$, Tianshi $\mathrm{Lu}^{2}$, and Yarema Prykarpatskyy ${ }^{1}$ \\ 1 Center for Data Intensive Computing, \\ Brookhaven National Laboratory, Upton, NY 11973, USA \\ \{rosamu, yarpry\}@bnl.gov \\ 2 Department of Applied Mathematics and Statistics, \\ SUNY at Stony Brook, Stony Brook, NY 11794, USA \\ tlu@sunysb.edu
}

\begin{abstract}
We have studied two approaches to the modeling of bubbly and cavitating fluids. The first approach is based on the direct numerical simulation of gas bubbles using the interface tracking technique. The second one uses a homogeneous description of bubbly fluid properties. Two techniques are complementary and can be applied to resolve different spatial scales in simulations. Numerical simulations of the dynamics of linear and shock waves in bubbly fluids have been performed and compared with experiments and theoretical predictions. Two techniques are being applied to study hydrodynamic processes in liquid mercury targets for new generation accelerators.
\end{abstract}

\section{Introduction}

An accurate description of cavitation and wave propagation in cavitating and bubbly fluids is a key problem in modeling and simulation of hydrodynamic processes in a variety of applications ranging from marine engineering to high energy physics. The modeling of free surface flows imposes an additional complication on this multiscale problem.

The wave propagation in bubbly fluids have been studied using a variety of methods. Significant progress has been achieved using various homogeneous descriptions of multiphase systems (see for example 11213 15 and references therein). The Rayleigh-Plesset equation for the evolution of the average bubble size distribution has often been used as a dynamic closure for fluid dynamics equations. This allows to implicitly include many important physics effects in bubbly systems such as the drug, viscosity, and surface tension. Numerical simulations of such systems require relatively simple and computationally inexpensive numerical algorithms. Nevertheless, homogeneous models cannot capture all features of complex flow regimes and exhibit sometimes large discrepancies with experiments 13 even for systems of non-dissolvable gas bubbles. Homogeneous models are also not suitable for modeling phase transitions in bubbly fluids such as boiling and cavitation. 
A powerfull method for multiphase problems, direct numerical simulation, is based on techniques developed for free surface flows. Examples of numerical simulations of a single vapor bubble undergoing a phase transition on its surface are given in 9,14 . Systems of bubbles in fluids were modeled in [7] using the incompressible flow approximation for both fluid and vapor and a simplified version of the interface tracking. In this paper, we describe a direct numerical simulation method for systems of compressible bubbles in fluids using the method of front tracking. Our FronTier code is capable of tracking and resolving topological changes of a large number of fluid interfaces in 2D and 3D spaces. We present the simulation results of the wave dynamics of linear and shock waves in bubbly systems and compare them with classical experiments.

The direct numerical simulations of wave dynamics in bubbly fluids in large 3D domains remain, however, prohibitively expensive even on supercomputers. Homogeneous models can effectively be used for such systems, especially if the resolving of spatial scales smaller then the distance between bubbles is not necessary. To model cavitating and bubbly fluids within the homogeneous approximation, we have recently developed and implemented in the FronTier code a two-phase equation of state (EOS) model based on the isentropic approximation. Therefore both direct and homogeneous approaches have advantages and disadvantages and can be used to resolve different temporal and spatial scales in numerical simulations.

Two numerical approaches are being used to study hydrodynamic processes involving cavitation and bubble dynamics in liquid mercury targets for new generation accelerators such as the Spallation Neutron Source (SNS) and the Muon Collider/Neutrino Factory (MC). Hydrodynamic insbabilities and cavitation in the MC mercury jet target will create complications for the machine operation. The collapse of cavitation bubbles in the SNS mercury target, resulting in the pitting of steel walls, has been the most critical problem reducing the target lifetime. The injection of layers of gas bubbles in mercury has been proposed as a possible pressure mitigation technique. These processes must be studied by means of large-scale numerical simulations.

The paper is organized as follows. In Section 2, we describe briefly the direct and homogeneous methods for the modeling of bubbly flows. Section 3 contains results of the numerical simulation of linear and shock waves in bubbly liquids using the direct and homogeneous techniques. We discuss classical shock tube experiments and applications to liquid mercury targets. Finally, we conclude the paper with a summary of our results and perspectives for future work.

\section{Modeling of Multiphase Flows}

\subsection{Direct Method}

In the direct method, we model a liquid - vapor or liquid - non-dissolvable gas mixture as a system of one phase domains separated by free interfaces. The FronTier code represents interfaces as lower dimensional meshes moving through a 
volume filling grid 4,5]. The traditional volume filling finite difference grid supports smooth solutions located in the region between interfaces. The dynamics of the interface comes from the mathematical theory of Riemann solutions, which are idealized solutions of single jump discontinuities for a conservation law. The FronTier code is capable of tracking simultaneously a large number of interfaces and resolving their topological changes (the breakup and merger of droplets) in two- and three- dimensional spaces. Away from interfaces, FronTier uses high resolution hyperbolic techniques. Different equation of state models are used for gas/vapor bubbles and the ambient liquid. Though computationally intensive, such an approach is very accurate in treating important effects in bubbly flows including phase transitions in boiling and cavitating fluids. The method makes it possible to resolve spatial scales smaller than the typical distance between bubbles and to model some non-equilibrium thermodynamics features such as finite critical tension in cavitating liquids.

\subsection{Homogeneous Method}

The advantage of the homogeneous method compared to the direct one is its computational simplicity and, as a result, low computational requirements for the simulation of large systems. The homogeneous flow approximation provides a simple technique for analyzing two-phase (or multiple phase) flows. It is accurate enough to handle a variety of practically important processes. Suitable averaging is performed over the length scale which is large compared to the distance between bubbles and the mixture is treated as a pseudofluid that obeys an equation of state (EOS) of a single component flow [15].

We have recently developed 11] a simple isentropic homogeneous equation of state for two-phase liquids and implemented the corresponding software library in the FronTier code. The isentropic approximation reduces by one the number on independent variables defining the thermodynamic state. As a result, all thermodynamic states in our EOS are functions of only density. The proposed EOS consists of three branches. The pure vapor and liquid branches are described by the polytropic and stiffened polytropic [10] EOS models, respectively, reduced to a single isentrope. The two branches are connected by a model for the liquid-vapor mixture

$$
P=P_{s a t, l}+P_{v l} \log \left[\frac{\rho_{\text {sat }, v} a_{s a t, v}{ }^{2}\left(\rho_{\text {sat }, l}+\alpha\left(\rho_{\text {sat }, v}-\rho_{\text {sat }, l}\right)\right)}{\rho_{\text {sat }, l}\left(\rho_{\text {sat }, v} a_{\text {sat }, v^{2}}-\alpha\left(\rho_{\text {sat }, v} a_{s a t, v^{2}}-\rho_{\text {sat }, l} a_{\text {sat }, l^{2}}\right)\right)}\right],
$$

where $\rho_{s a t, v}, \rho_{s a t, l}, a_{\text {sat }, v}, a_{\text {sat }, l}$ are the density and the speed of sound of vapor and liquid in saturation points, respectively, $P_{s a t, l}$ is the liquid pressure in the saturation point, $\alpha$ is the void fraction

$$
\alpha=\frac{\rho-\rho_{\text {sat }, l}}{\rho_{\text {sat }, v}-\rho_{\text {sat }, l}},
$$

and the parameter $P_{v l}$ is

$$
P_{v l}=\frac{\rho_{s a t, v} a_{s a t, v}{ }^{2} \rho_{s a t, l} a_{s a t, l^{2}}\left(\rho_{s a t, v}-\rho_{s a t, l}\right)}{\rho_{s a t, v^{2}} a_{s a t, v^{2}}-\rho_{s a t, l^{2}} a_{s a t, l^{2}}} .
$$


These expressions were derived by integrating an experimentally validated model for the sound speed in bubbly mixture [15]. A set of the EOS input parameters most of which are measurable quantities allows to fit the two-phase EOS to thermodynamics data for real fluids. Details on the EOS model are presented in 11.

The FronTier code with two-phase EOS modes has been applied to study the interaction of mercury with an intensive proton pulse in the geometry typical for mercury thimble neutrino factory experiments. The use of two-phase EOS has led to improvement over single phase EOS simulations [12] of the mercury surface evolution.

\section{$3 \quad$ Numerical Results}

\subsection{Direct Numerical Simulations}

In this section, we present results of the direct numerical simulation of the dynamics of linear and nonlinear waves in bubbly liquids. The schematic of the numerical experiment setup is depicted in Figure 1. The liquid contains nondissolvable gas bubbles at normal conditions. The bubble radius is $0.12 \mathrm{~mm}$ and the void fraction is $1.55 \cdot 10^{-4}$. The region around a long column of bubbles was chosen as the computational domain. As the first order approximation, we can assume that the pressure waves are axisymmetric. The influence of neighboring bubbles can be effectively approximated by the Neumann boundary condition on the domain walls. Therefore the wave propagation in bubbly flows was reduced to an axisymmetric two-dimensional problem.

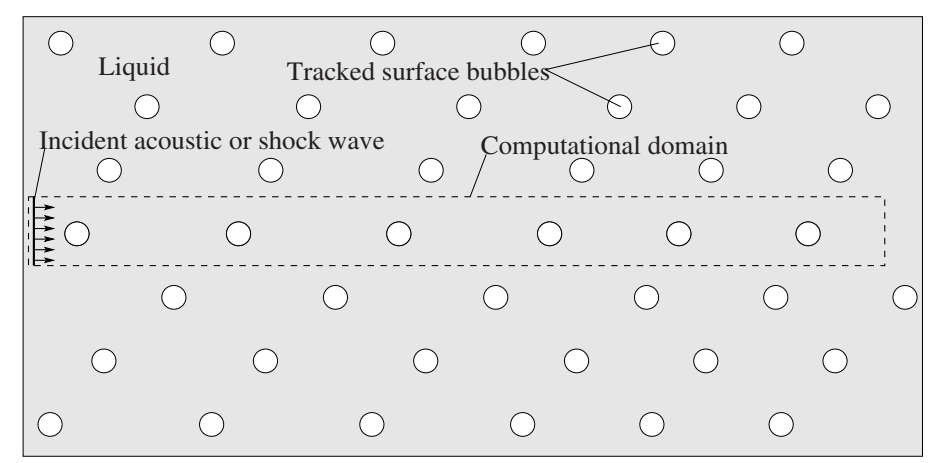

Fig. 1. Schematic of the numerical experiment. The computational domain contained 100 bubbles

Our first numerical experiments were performed with small amplitude linear waves in bubbly fluids. Measuring the dispersion relation and the attenuation rates from simulations, we found that results are in good agreement with Fox, 
Curley and Larson's experiments [3] as well as theoretical predictions [15]. The numerical and theoretical results are depictured in Figure 2.

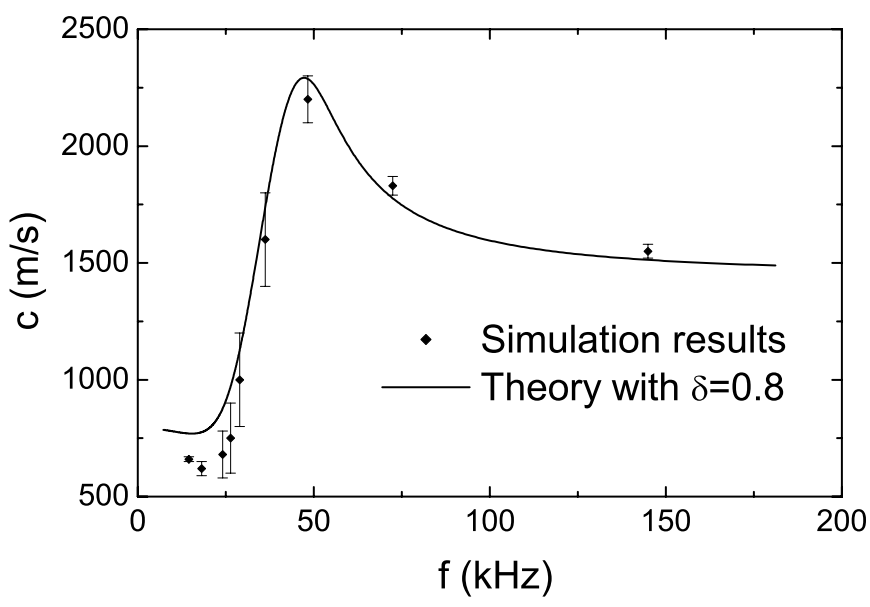

Fig. 2. Dispersion relation in bubbly flows. The bubble radius is $0.12 \mathrm{~mm}$, void fraction is $1.55 \cdot 10^{-4}$, and the pressure is 1.0 bar. The amplitude of the incident pressure wave is 0.1 bar. Grid size is $90 \times 10800, \delta$ is the damping coefficient

Figure 3 depicts results of experiments and numerical simulations of the interaction of shock waves with bubbly layers. We measured shock velocities and plotted shock profiles for bubbles consisting of different kinds of gases. The shock speeds agreed with the theoretical prediction of [13] very well (with the difference less than 3\%). The shock profiles agreed with experiments of Beylich and Gulhan [1] qualitatively and partly quantitatively. Some discrepancy in the amplitude of pressure oscillations can be explained by grid related numerical errors. To improve the accuracy and performance of the direct method, we have been working on the adaptive mesh refinement method for the FronTier code. Current simulations performed on $90 \times 10800$ grids required several days of CPU time on a parallel cluster of Pentium processors. Both simulations and experiments showed that the amplitude of pressure ocsillations in the bubbly layer after the passage of the shock front is smaller for the gas with larger polytropic index.

We have also performed preliminary numerical simulations of the interaction of bubbly mercury with a strong proton pulse in the SNS target. The use of layers of gas bubbles has been proposed as a pressure mitigation technique which may reduce the cavitation induced erosion and extend the target lifetime. Preliminary results confirmed the usefulness of this mitigation method. 

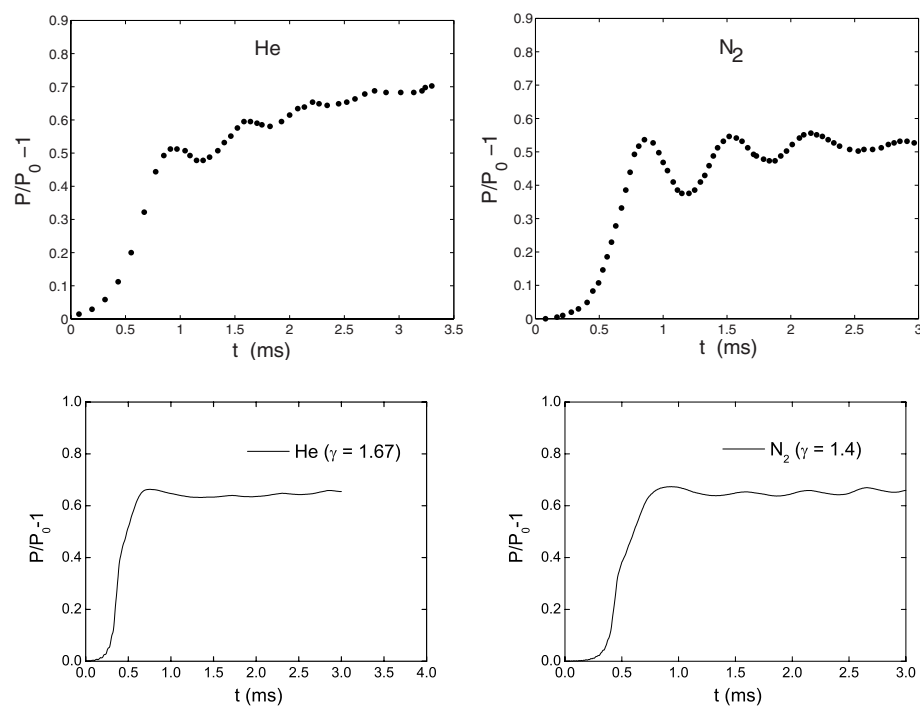

Fig. 3. Experiments of Beylich and Gulhan 11 (top) and FronTier numerical simulations (bottom) of shock waves in bubbly liquids. The bubble radius is $1.18 \mathrm{~mm}$, the void fraction is $2.5 \cdot 10^{-3}$, the fluid pressure is $1.1 \mathrm{bar}$, and the initial shock pressure is 1.727 bar. Grid size is $35 \times 7000$. Shock profiles were measured $40 \mathrm{~cm}$ from the interface

\subsection{Numerical Simulation of Interaction of Mercury with Protons Using the Homogeneous Model}

In this section, we present numerical simulation results of the interaction of free surface mercury with strong proton pulses using FronTier code with the homogeneous two-phase EOS model. To study the influence of proton pulse induced thermal shocks on mercury targets, a series of experiments were conducted at the Alternating Gradient Synchrotron (AGS) at BNL and On-Line Isotope Mass Separator facility (ISOLDE) at CERN [6]8]. We will discuss here some experimental and numerical simulation results of the mercury thimble studies.

The volume of the thimble excavated in a stainless steel bar is $1.3 \mathrm{~cm}^{3}$. It consists from bottom to top of a half sphere $(\mathrm{r}=6 \mathrm{~mm})$, and a vertical cylinder $(\mathrm{r}=\mathrm{h}=6 \mathrm{~mm})$. The mercury has a free surface in up-direction, where it can expand to. The mercury interacts with a proton pulse of approximately Gaussian distribution. The pulse intensity range is $0.6-17 \cdot 10^{12}$ protons at energy $24 \mathrm{GeV}$.

We have performed numerical simulations of the mercury splash evolution in the thimble using the FronTier code with the two-phase EOS (Figure 5). We have studied the evolution of the mercury splash in the thimble at different values of the proton intensity and the r.m.s. spot size of the beam, and obtained a good agreement with experimental data. However, numerical simulation do not capture some experimentally observable fine effects in the splash evolution such as the reduction of the splash velocity during first 2 microseconds after 
the arrival of the proton pulse 6]. This discrepancy can be explained by the incomplete thermodynamics of our EOS. In the future, these simulations will also be performed using the direct method with full thermodynamics and the phase transition support.

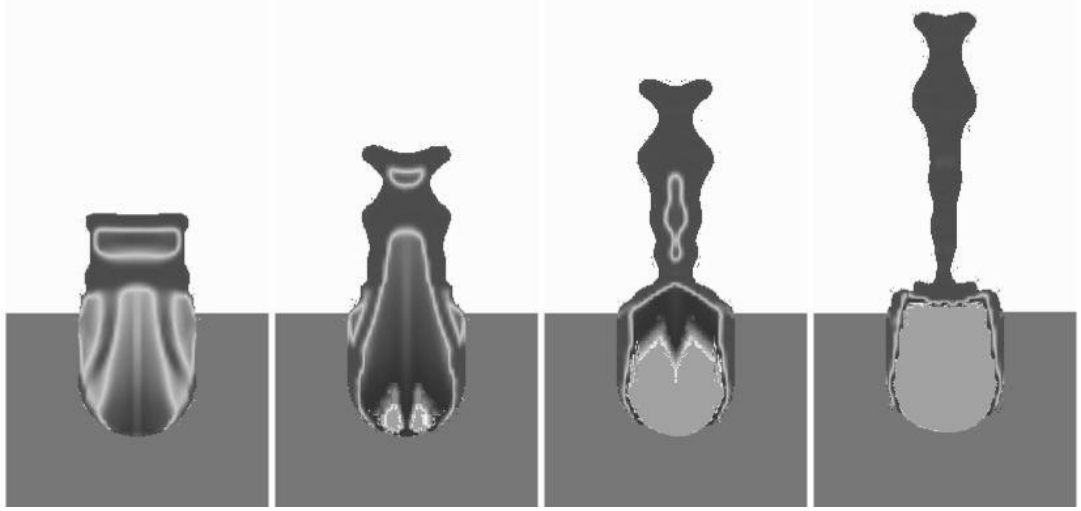

Fig. 4. Numerical simulation of the mercury splash in the thimble

\section{Conclusions}

We have developed the direct and homogeneous approaches within the FronTier code for the simulation of bubbly fluids. Through the comparison of numerical simulations with experiments and theoretical predictions on the propagation of linear and shock waves in bubbly fluids, the direct approach which uses the method of front tracking and the FronTier code has been validated. The direct method has a variety of current and potential applications. It is being applied for numerical simulations of the interaction of bubbly mercury with strong proton pulses in the SNS target. The use of layers of gas bubbles has been proposed as a pressure mitigation technique which may reduce the cavitation induced erosion and extend the target lifetime. Preliminary simulations have demonstrated an effective reduction of the peak pressure. The mass transfer across bubble surfaces due to the phase transition is being implemented in the code. This will allow to simulate systems of vapor bubbles in boiling and cavitating liquids. To improve the numerical resolution and performance of the code, an adaptive mesh refinement method will be used in future simulations.

To model cavitating and bubbly fluids within the homogeneous approximation, we have developed and implemented in the FronTier code a two-phase equation of state (EOS) model based on the isentropic approximation. This approach is especially suitable if spatial scales smaller than the distance between bubbles can be neglected for global studies of the wave dynamics. It requires 
coarser grids compared to the direct numerical simulation method and often significantly smaller CPU time. The code has been applied to study the interaction of mercury with an intensive proton pulse in the geometry typical for mercury thimble neutrino factory experiments. The simulations are in good quantitative agreement with experiments. The direct and homogeneous approaches are complementary and can be used to resolve different temporal and spatial scales in numerical simulations.

Acknowledgments. The authors are grateful to James Glimm, Xiaolin Li, and Harold Kirk for fruitful discussions. Financial support has been provided by the USA Department of Energy, under contract number DE-AC02-98CH10886.

\section{References}

1. Beylich, A.E., Gülhan, A.: On the structure of nonlinear waves in liquids with gas bubbles. Phys. Fluids A 2 (1990) $1412-1428$

2. Caflisch, R.E., Miksis, M.J., Papanicolaou, G.C., Ting, L.: Effective equations for wave propagation in bubbly liquids. J. Fluid Mech. 153 (1985) $259-273$

3. Fox, F.E., Curley, S.R., Larson,G.S.: J. Acoust. Soc. Am. 27 (1955) 534

4. Glimm, J., Grove, J.W., Li, X.L., Shyue, K.M., Zhang, Q., Zeng, Y.: Three dimensional front tracking. SIAM J. Sci. Comp. 19 (1998) $703-727$

5. Glimm J., Grove J., Li X.-L, and Tan D.C.: Robust computational algorithms for dynamic interface tracking in three dimensions. SIAM J. Sci. Comp. 21 (2000) $2240-2256$

6. Fabich, A., Lettry J.: Experimental Observation of Proton-Induced Shocks and Magneto-Fluid-Dynamics in Liquid Metal. In Proceedings NuFact 01, NIM A, (2001)

7. Juric, D., Tryggvason, G.: Computation of boiling flows. Int. J. Multiphase Flow 24 (1998) $387-410$

8. Kirk, H., et al.: Target studies with BNL E951 at the AGS. Particles and Accelerators 2001, June 18-22 (2001) Chicago IL

9. Matsumoto, Y., Takemura, F.: Influence of internal phenomena on gas bubble motion. JSME Int. J. 37 (1994) $288-296$

10. Menikoff, R., Plohr, B.: The Riemann problem for fluid flow of real materials. Rev. Mod. Phys. 61 (1989) $75-130$

11. Samulyak, R., Prykarpatskyy, Y.: Richtmyer-Meshkov instability in liquid metal flows: influence of cavitation and magnetic fields. Mathematics and Computers in Simulations (2003). In press

12. Samulyak, R.: Numerical simulation of hydro- and magnetohydrodynamic processes in the Muon Collider target. Lecture Notes in Comp. Sci., Vol. 2331. SpringerVerlag, Berlin Heidelberg New York (2002) 391 - 400

13. Watanabe, M., Prosperetti, A.: Shock waves in dilute bubbly liquids. J. Fluid Mech. 274 (1994) $349-381$

14. Welch, S.W.: Local simulation of two-phase flows including interface tracking with mass transfer. J. Comp. Phys. 121 (1995) $142-154$

15. Wijngaarden, L. Van: One-dimensional flow of liquids containing small gas bubbles. Ann. Rev. Fluid Mech. 4 (1972) 369-396 